\title{
A place for pot in sports?
}

Cite as: CMAJ 2017 March 20;189:E448-9. doi: 10.1503/cmaj.1095401

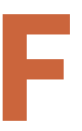

or professional athletes, dealing with pain is an everyday problem, even after they retire. Torn ligaments, dislocated shoulders, busted knees and other injuries are part of the job and tend to have a lasting impact. Something that isn't so common in sports is medical marijuana, which remains off limits in some leagues, even though it doesn't make athletes stronger or faster or enhance performance in any other way. Some athletes, however, are challenging old attitudes about cannabis, suggesting it is a safer alternative to managing pain than the drugs commonly used in sports.

In Canada, the Canadian Centre for Ethics in Sports manages an anti-doping program, and sports organizations that are signatories to the program can't allow their athletes to use drugs on a prohibited list. The list was created by the World AntiDoping Agency and it includes marijuana.

"If an athlete has a medical condition that requires them to take a specific medication (such as marijuana) that appears on the prohibited list, they can apply for a medical exemption," the centre said in a statement. "We aren't able to comment on the anti-doping policies of professional leagues, but ideally, we would like to see professional sports adopt codecompliant anti-doping programs."

It should come as no surprise that contact sports have higher injury rates, and there is perhaps no professional sport with more bone-jarring contact that football. For example, in the 2015 season of the National Football League (NFL), it took only two weeks for $15 \%$ of all players to suffer an injury.

Some former NFL players recently participated in an event called "Cannabis in Professional Sports" in hopes of changing attitudes about using marijuana to manage pain in professional football. Some of the players said the injuries they sustained during their athletic careers mean they now live in chronic pain. One former player said he couldn't even bend over to pick up his children.

To deal with the pain, some used alcohol or dangerous illicit drugs, such as heroin, before turning to cannabis. Public endorsement of medical marijuana remains taboo among current NFL players, however, because taking a stand could cost them their jobs.

The NFL isn't the only American sports league that bans marijuana. The National Basketball Association and Major League Baseball also prohibit the drug. Canadian sports leagues tend to be more lax in this area. The Canadian Football League doesn't test for marijuana. A third of National Hockey League players are randomly tested for street drugs, including marijuana, but they aren't disciplined for positive tests.
Liberal use of prescription drugs, however, is common in most professional sports. It is well known that the need to manage pain in the NFL has led to a culture of drug use and abuse. To remain on the field, many players take large quantities of painkillers and anti-inflammatories. Using pills and injections is as commonplace as attending practices and taking ice baths.

A survey of former NFL players found that $52 \%$ had used opioids during their playing days, and the rate of opioid use among retired players was triple that of the general population. The NFL Players Association, which represents the interests of the athletes, has formed a pain management committee to explore using marijuana as a treatment for pain, and has implored the league to take a less punitive stance on the use of cannabis.

Producers of medical marijuana are optimistic that the drug will eventually be seen as safer alternative to pharmaceutical-

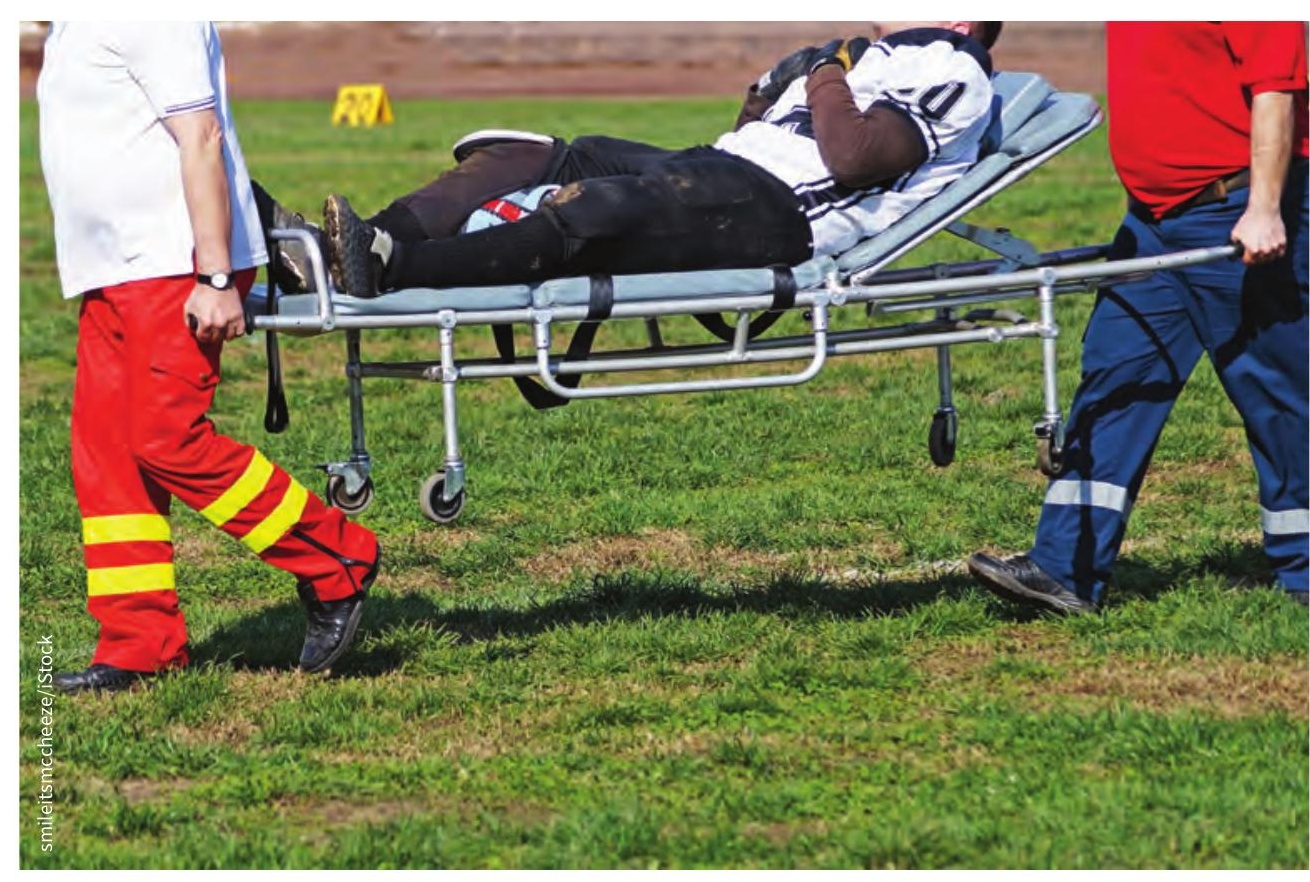

Injuries - and pain - are inevitable in contact sports such as football. 
based pain management, not only in sports but all of society. "Although human studies on the therapeutic effects of cannabis have been significantly limited to date (largely due to restrictive legal regimes), anecdotal patient reports suggest that opportunities for the use of medical cannabis in the treatment of chronic pain are significant," said Brent Zettl, president and CEO of CanniMed Therapeutics Inc. in Saskatchewan.
Some cannabis producers are funding research on pain management to fill the void, including British Columbia-based Tilray. The company funded a survey recently published in the International Journal of Drug Policy on substituting cannabis for prescription opioids and other substances. "There is a growing amount of evidence suggesting that cannabis is a safer substitute for many prescription drugs, particularly opioids," said Philippe Lucas, vice president of patient research and access at Tilray. "Increasing the understanding and acceptance of medical cannabis as a relatively safe and effective treatment for chronic pain and other conditions could reduce the medical community's dependence on opioids."

Roger Collier, CMAJ 